\title{
Improved Image Denoising Using Wavelet Edge Detection Based on Otsu's Thresholding
}

\section{Tuğba Özge Onur}

Zonguldak Bülent Ecevit University, Dept. of Electrical-Electronics Engineering, 67100 Zonguldak, Turkey

E-mail: tozge.ozdinc@ @eun.edu.tr

\begin{abstract}
Images are affected by noise during their acquisition and transmission. Therefore, the denoising process is necessary to achieve higher quality images. However, both edges of the image and noise are characterized by high frequencies, loss of edge information may become unavoidable as a result of the denoising process. Thus, recovered, denoised images, become blurrier or less denoised. Therefore, a wavelet threshold denoising technique, based on edge detection, can be used to preserve more edge information and enhance the quality of the denoised image. In this paper, a novel image denoising method, based on wavelet thresholding by using Otsu's threshold, has been proposed and the clarity of the image which has been handled with this method is superior to that currently achieved by the other wavelet thresholds. The obtained results show that the proposed method, in this paper, provides better performance compared to commonly used wavelet image threshold denoising methods in terms of the visual quality of the denoised image. In addition, when the edge detection and denoising processes are combined, the deficiencies of the commonly used denoising methods are eliminated and a better denoising effect has been achieved.
\end{abstract}

Keywords: wavelet threshold; image denoising; wavelet edge detection; peak signal to noise ratio (PSNR)

\section{Introduction}

Image denoising is one of the basic tasks for the researchers dealing with image processing since there may occur distortions of images during the acquisition, processing, compression, transmission or reconstruction processes. Therefore, it is important to eliminate the noise from the images and increase the quality, or produce good estimates from noisy ones. The image noise can be Gauss, Poisson, or particle noise [1] [2]. The visuality and processing of the image are both affected by the noise. Therefore, it is aimed to preserve the useful information of the image and to reduce the noise by the image denoising process.

Since denoising is a preliminary process in the field of image processing, almost all researchers interested in image processing have dealt with this problem and 
therefore researches on this effect made significant progress. Spectrum distribution is used for the traditional image denoising algorithms. In other respects, there have been various methods including the Markov field model, neural networks, nonlinear diffusion, 3-dimensional block-matching, etc. to remove the noise from the images [3]. Although there are a wide variety of methods for the image denoising process, there have been challenges for most of the current denoising techniques because by the use of these techniques high frequencies can be suppressed. Thus, since noise can be characterized by high frequencies, high accurate results may not be achieved by the use of these techniques. Therefore, wavelet transform has been used extensively for various applications such as denoising in signal and image processing since there have been drawbacks of noise regularizing for other methods. In addition, successful results are achieved in applications due to an easily applicable algorithm and significant noise reduction effect of the wavelet transform. Due to its potential in the signal denoising process, its use has received significant attention from researchers. As more timely topics, the researchers have been attracted.

\subsection{Related Works and Motivation}

Although there have been various studies related to image denoising in the literature, researchers are still dealing with novel algorithms which have easy-toimplement applications. Lee et al. have presented a nonlinear diffusion filtering method and tried to improve the denoising process [4]. Mallat and Hwang [5] proposed an alternating projection method in 1992 and Zhu et al. [6] improved this method by obtaining the modulus maxima at each scale in 2017. In 2006, a denoising method based on sparse representation was used by Elad and Aharon [7]. Furthermore, artificial neural network models have been presented for the filtering processes of the noisy images [6] [7]. Moreover, fuzzy models can be used in noise image processing. Minh and Chen presented a generalized fuzzy system and achieved high performance for noise modeling in images [8]. A recurrent intervalvalued fuzzy neural network was proposed by Juang et al. in 2011 [9]. Cheng and Juang proposed a fuzzy model that is based on support vector machine and margin selected gradient descent learning [10]. In addition, there have been several studies related to determining the efficient band by using a canonical correlation classifier. For example, Pozna and Precup suggested a new approach to model the system and represent the data with signatures [11].

Zall and Kangavari introduced an approach that was based on canonical correlation analysis and extracted correlation information between the significant paths [12]. Apart from these, denoising methods based on machine learning including k-nearest neighbor regression, etc. have been used for some researches [13]-[17]. Borlea et al. proposed a clustering algorithm that processes data sets of any size. They used it for the Iris dataset however suggested modifying it for different datasets [18]. 
Furthermore, not only due to the drawbacks of these methods but also the low performance of the classifier or model-based approaches in the literature, waveletbased denoising methods can be available. Johnstone and Silverman [19] and Othman and Qian [20] have used wavelet transformation to distinguish useful information and noise from images. In addition, in recent studies, Bnou et al. have presented a new wavelet denoising method that uses an unsupervised learning model [21]. However, supervised learning models require prior learning of the corrupted image. Therefore, a thresholding filter is widely used to implement the denoising algorithm in the wavelet domain [22] [23]. Since the signal is transformed from the time domain to the time-scale domain in wavelet transform, a threshold value can be selected easily to reduce the noise. However, it is important to determine the appropriate value for the threshold, higher values result in better denoising whereas causes blurred edges. This is an undesirable result. Since the edges are mostly contain the information and basic character of the image, in some cases, loss or corruption of this information causes erroneous results [24]. Therefore, this results in data losses for the edges' knowledge since edges are characterized by high frequencies like noise, too. Donoho and Johnston [22] [24], [25] proposed hard and soft thresholding methods depending on the noise power and image size for denoising. In addition, other wavelet-based thresholding methods such as VisuShrink, Oracle Shrink, Normal Shrink have been used to obtain efficiency results in image denoising [26]. Thereafter, a wavelet edge detection based on both VisuShrink and scaled VisuShrink thresholding method was proposed by Liu and Ma and they proved that it outperforms classical wavelet thresholding methods [27]. Recently, machine learning is combined with traditional denoising methods [28]. However, the thresholding in denoising in images is still a crucial task and subject of research.

In recent researches, it has become even more important to reach the details of the images and eliminate the noise from them. In this paper, new wavelet thresholding to image denoising based on Otsu's thresholding is presented.

\subsection{Contribution}

The main contributions of this paper are as follows:

- The wavelet edge detection is used to detect the wavelet coefficients for the edges in the image before the denoising process by combining Otsu's thresholding which is one of the classical thresholding methods.

- Since the thresholds for the edges are set by Otsu's method, the wavelet coefficients can be thresholded without damaging the edges.

- The novelty of the proposed algorithm is the use of Otsu's thresholding and not to require any information concerning the noise level of the image. In addition, this is also the first study that combines Otsu's thresholding and wavelet transform algorithms to the best of the authors' knowledge. 
- The extensive simulations are presented to validate the robustness and accuracy of the proposed denoising method. Based on the simulation results, it is revealed that the proposed method in this study is more effective compared with the other wavelet threshold denoising methods and it allows to achieve increasing denoising performances for the noisy images.

\subsection{Organization}

The rest of this paper is organized as follows. Section 2 describes the wavelet image edge detection. The proposed method in this paper is explained in detail in Section 3. Section 4 presents the simulation results to compare the performance of the method with related ones performed in recent years. Finally, the results are discussed and the paper is concluded in Section 5.

\section{The Wavelet Image Edge Detection Method}

In wavelet edge detection, a 2-dimensioned (2-D) wavelet transformation is required [9]. Therefore, two wavelets are needed as given in Eq. (1) to perform this transform.

$$
\emptyset_{2^{j}}^{x}(x, y)=\frac{\partial \theta_{2^{j}}(x, y)}{\partial x}, \quad \emptyset_{2^{j}}^{y}(x, y)=\frac{\partial \theta_{2^{j}}(x, y)}{\partial y}
$$

where $\emptyset_{2^{j}}^{x}(x, y)$ and $\emptyset_{2^{j}}^{y}(x, y)$ are the wavelets and $\theta_{2^{j}}(x, y)$ is a smoothing function. The wavelet coefficients of an image $g(x, y)$ can be determined as given in Eq. (2):

$$
\begin{aligned}
{\left[\begin{array}{l}
W_{2^{j}}^{x} g(x, y) \\
W_{2^{j}}^{y} g(x, y)
\end{array}\right] } & =\left[\begin{array}{l}
g^{*} \emptyset_{2^{j}}^{x}(x, y) \\
g^{*} \emptyset_{2^{j}}^{y}(x, y)
\end{array}\right] \\
& =2^{j}\left[\begin{array}{c}
\frac{\partial}{\partial x} g^{*} \theta_{2^{j}}(x, y) \\
\frac{\partial}{\partial y} g^{*} \theta_{2^{j}}(x, y)
\end{array}\right]=2^{j} \nabla\left(g^{*} \theta_{2^{j}}\right)(x, y)
\end{aligned}
$$

where $2^{j}$ is a scale factor. By considering Eq. (2), the edges can be detected with the scale factor $2^{j}$ since they can be explained by the local maxima of the gradient. If the image is distorted by additive noise, there may be some other pixels in the image which have a local maximum of the gradient. Therefore, it is important to be able to determine the coefficients belonging to the noise and the edges to prevent any loss of information in the denoising process. This distinction between the noise and the edges is made by considering Lipschitz exponent values if the additive noise in the image is the additive white Gaussian noise (AWGN) [29]. While applying 
edge detection in the wavelet edge detection method, the image is an average filtered with appropriate length. For the second step, the initial is assumed to be $S_{2} \circ g(x, y)=g(x, y)$ and wavelet transformation is applied to each row of $S_{2^{j}} g(x, y)$. Therefore, the used filters $G_{j}$ and $H_{j}$ in discrete wavelet transform (DWT) can be calculated as given in Eq. (3):

$$
\begin{aligned}
& W_{2^{j+1}}^{y} g=\left(S_{2^{j}} g\right) * G_{j} \\
& S_{2^{j+1}} g=\left(S_{2^{j}} g\right) * H_{j} \quad \mathrm{j}=0,1,2, \ldots
\end{aligned}
$$

Here, the scale is represented by $2^{j}$. For every row, coefficients of local maximum are found and saved in $W_{2^{j}}^{y} g(x, y)$. Since noise has low Lipschitz exponent values, the coefficients with these low values are removed in $W_{2^{j}}^{y} g(x, y)$. By applying this process, the remain coefficients in $W_{2^{j}}^{y} g(x, y)$ correspond to the edges of each row. If the same procedures are applied for each column, the coefficients of edges in $W_{2^{j}}^{x} g(x, y)$ can be obtained. Finally, a threshold value is determined by using these wavelet coefficients. However, Liu and Ma have proposed a wavelet edge detection method to overcome the problem of thresholding the wavelet coefficients with the appropriate threshold value. They used the wavelet edge detection method to detect the edges of the image before the denoising process. So, they set thresholds based on noise variances by using the VisuShrink threshold and since wavelet coefficients are protected, the information of the edges is not damaged [27]. However, determining the strength of noise in this thresholding may pose a problem for the images that have an indefinite noise level.

\section{The Proposed Denoising Method: The Combination of Otsu's Thresholding and Wavelet Edge Detection}

In the wavelet edge detection method, it is important to determine the appropriate threshold value while thresholding wavelet coefficients because noises are not clustered in a few wavelet coefficients. Therefore, if the threshold is not chosen high enough, the noise may not be reduced significantly. On the other hand, if it has a higher value, the better denoising performance will occur however it will result in blurred edges. So, to overcome this challenge, in the proposed method in this paper, the edges of the images are determined by the wavelet edge detection method in the first stage, and thereafter Otsu's thresholding is used to execute the wavelet threshold denoising process. The novelty of this study is to use Otsu's thresholding instead of thresholds which are based on the noise variances such as VisuShrink, Oracle Shrink, Normal Shrink thresholds, etc. The reason for using Otsu's thresholding is that Otsu's thresholding maximizes the between-class variance [30]. 


\subsection{Otsu's Threshold Method}

It is performed by selecting the lowest point between two classes. Therefore, edges can be determined without identifying the strength of the noise. Equations (4)-(8) describe the theoretical background of Otsu's thresholding.

$$
\begin{aligned}
& \sigma^{2}=\sigma_{w}^{2}(t)+q_{1}(t)\left[1-q_{1}(t)\right]\left[\mu_{1}(t)-\mu_{2}(t)\right]^{2} \\
& \sigma_{w}^{2}(t)=q_{1}(t) \sigma_{1}^{2}(t)+q_{2}(t) \sigma_{2}^{2}(t) \\
& q_{1}(t)=\sum_{i=1}^{t} P(i), q_{2}(t)=\sum_{i=t+1}^{I} P(i) \\
& \mu_{1}(t)=\sum_{i=1}^{t} \frac{i P(i)}{q_{1}(t)}, \mu_{2}(t)=\sum_{i=t+1}^{I} \frac{i P(i)}{q_{2}(t)} \\
& \sigma_{1}^{2}(t)=\sum_{i=1}^{t}\left[i-\mu_{1}(t)\right]^{2} \frac{P(i)}{q_{1}(t)}, \sigma_{2}^{2}(t)=\sum_{i=t+1}^{I}\left[i-\mu_{2}(t)\right]^{2} \frac{P(i)}{q_{2}(t)}
\end{aligned}
$$

In Eqs. (4)-(8), $\sigma_{w}^{2}(t)$ is the weighted within-class variance, $q_{1}(t)$ and $q_{2}(t)$ are the probabilities of the classes, $\mu_{1}(t)$ and $\mu_{2}(t)$ are the means of the classes, $\sigma_{1}^{2}(t)$ and $\sigma_{2}^{2}(t)$ are the variances of individual classes [30].

\subsection{The Proposed Image Denoising Approach}

In the proposed image denoising method in this paper, firstly, the wavelet coefficients that correspond to the edges of the image are determined by using the wavelet edge detection method which is detailed in Section 2 with Eqs. (1)-(3). Thereafter, these coefficients are preserved and wavelet transform is performed to the distorted image by noise. As the third stage, the wavelet image threshold denoising process is applied by using Otsu's thresholding as defined in Eq. (9):

$$
\widetilde{\mathrm{w}}= \begin{cases}\mathrm{w} & |\mathrm{w}| \geq \mathrm{T} \\ 0 & |\mathrm{w}|<T\end{cases}
$$

where T represents Otsu's threshold value. In the fourth stage, the coefficients which correspond to the image edges are replaced with the coefficients. In case the determined edges may also include noise, it is again thresholded with $\alpha \mathrm{T}$ where $\alpha$ is an adjustment factor between 0.125 and 0.9. After the threshold $\mathrm{T}$ is determined, the $\alpha$ value is chosen to provide a better peak signal-to-noise ratio (PSNR). To determine the appropriate $\alpha$ value, some trials are performed by investigating the PSNR values and the results are shown in Table 1. The PSNR is defined as given in Eq. (10): 


$$
P S N R=10 * \log \left(\frac{255^{2}}{\frac{1}{M * N}\left[\sum_{x=1}^{M} \sum_{j=1}^{M}((g(x, y)-\hat{g}(x, y)))^{2}\right]}\right)
$$

where $\mathrm{M} * \mathrm{~N}$ denotes the image size, $g(x, y)$ and $\hat{g}(x, y)$ are the original and the reconstructed denoised images, respectively.

Table 1

PSNR (dB) values for different $\alpha$ adjustment factors and $\sigma$ noise variances for $256 * 256$ Chairs image

$\begin{array}{lccccc}\begin{array}{l}\text { Noise } \\ \text { variance }\end{array} & \boldsymbol{\alpha}=\mathbf{0 . 1 2 5} & \boldsymbol{\alpha}=\mathbf{0 . 2 5} & \begin{array}{c}\boldsymbol{\alpha}=\mathbf{0 . 5} \\ \boldsymbol{\alpha}=\mathbf{0 . 9}\end{array} & \boldsymbol{\alpha}=\mathbf{1} \\ \sigma=5 & 33.2851 & 33.4257 & 31.0618 & 28.4731 & 28.1641 \\ \sigma=10 & 28.4524 & 28.5888 & 27.7786 & 26.5616 & 26.2353 \\ \sigma=15 & 24.9799 & 25.2984 & 25.2109 & 24.5371 & 24.4315 \\ \sigma=20 & 22.6096 & 22.9033 & 23.1415 & 22.7989 & 22.7463 \\ \sigma=25 & 20.7398 & 20.9612 & 21.4797 & 21.3800 & 21.2821 \\ \sigma=50 & 15.4561 & 11.4741 & 15.8323 & 16.4644 & 16.4117\end{array}$

It can be concluded from Table 1 that since noise is characterized by high frequencies like edges, in the case of the more disturbing image by noise $\alpha$ is chosen higher and otherwise smaller in the given range. Finally, the denoised image can be obtained by applying the inverse wavelet transform. These steps used in the proposed method are depicted with the flowchart in Fig. 1. In the proposed method given in Fig. 1, the wavelet coefficients which are the parameters of the model have been obtained by using Eqs. (1)-(3). Thereafter, the threshold value is determined with the Eqs. (4)-(8) and considering the adjustment factors and noise variances given in Table 1.

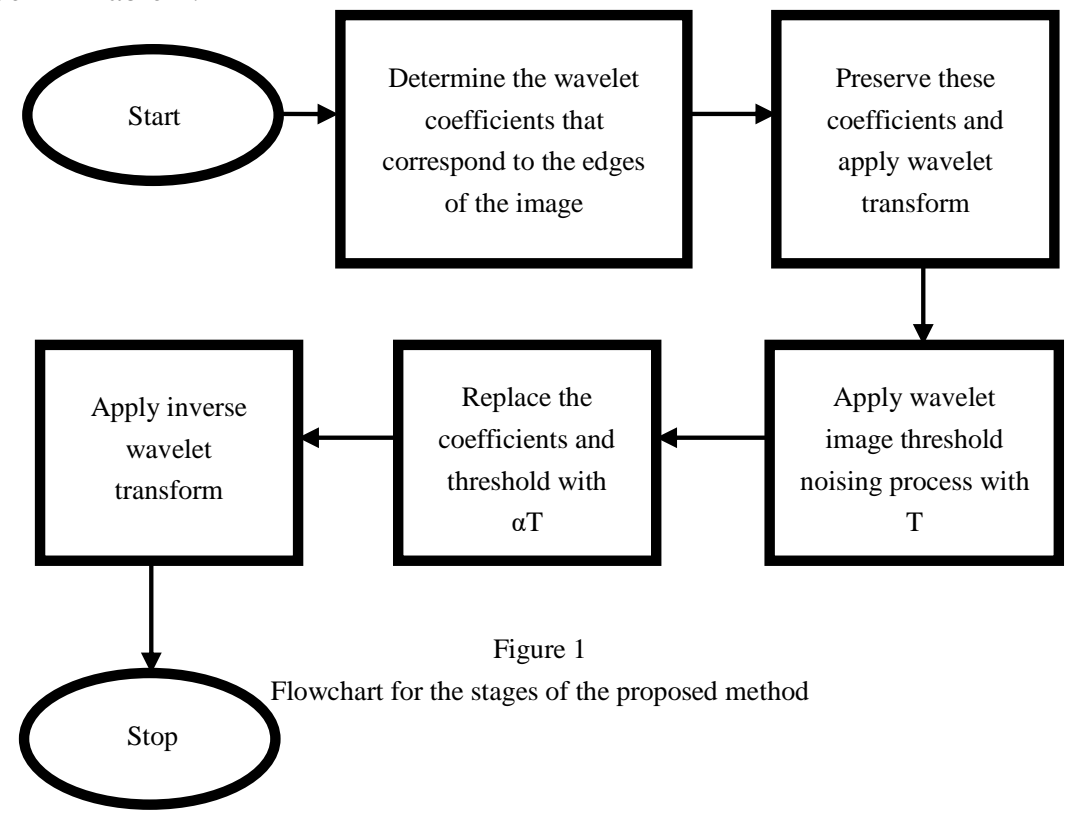




\section{Subjective Evaluation Results}

To evaluate the performance of the proposed method in this paper, Chairs image is preferred due to its different structured edges. This image is captured by a digital camera in Memorial Union which is a historical destination on UW-Madison's campus and resized in MATLAB 2018b program to provide convenience for the simulations. It is not easy to detect the different types of edges in the images completely by using a conventional edge detection method. In case of the image is corrupted by additive noise, this detection process will be even more difficult. For the simulations, the original $256 * 256$ Chairs image is distorted by AWGN with zero mean and different variances. Figure 2 and Figure 3 show the original Chairs image and distorted ones, respectively. Simulations are carried out in MATLAB 2018b. To prove the validity and effectiveness of the presented method, the performance comparison is made with the method which uses both VisuShrink and scaled VisuShrink thresholds suggested by Liu and Ma in Ref. 21. Results shown in Table 2 compare the proposed method in this paper and the method in which thresholding is performed by both $T=\sigma \sqrt{2 \ln N}$ (VisuShrink threshold) and $T=\beta \sigma \sqrt{2 \ln N}$ (scaled VisuShrink threshold). The noise variances are determined to be in the range of 5-50 and the value for the $\alpha$ adjustment factor is determined as $0.25,0.5,0.9$ for 5, 10, 15 and 20, 25 and 50 noise variances, respectively, by considering Table 1 .

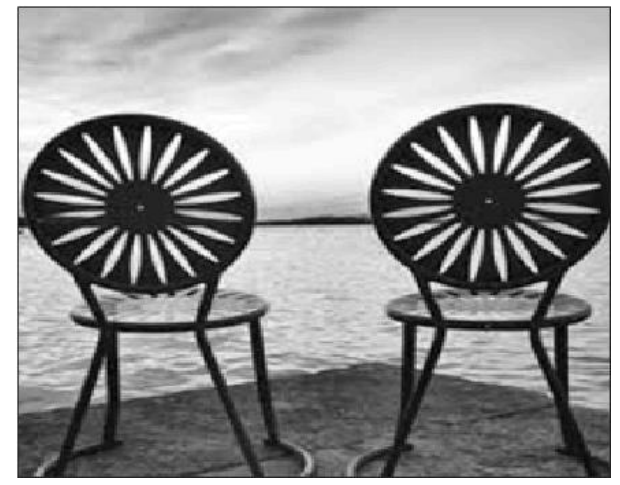

Figure 2

Original Chairs image

Figure 4 shows the obtained denoised images for different noise variances. In Fig. 4 , figures in the first, second, third, and fourth rows belong to the denoised ones of the noise distorted with the noise variances 5, 10, 15, 20, 25, and 50 respectively. In addition, the left and right columns of each line correspond to the obtained denoised images of the proposed methods in this paper (Otsu's thresholding) and VisuShrink and scaled VisuShrink thresholding, respectively. 


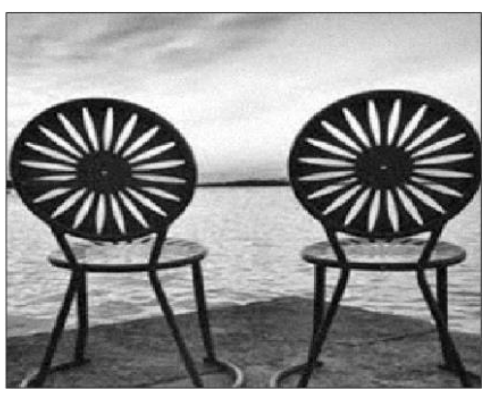

(a) $\sigma=5$

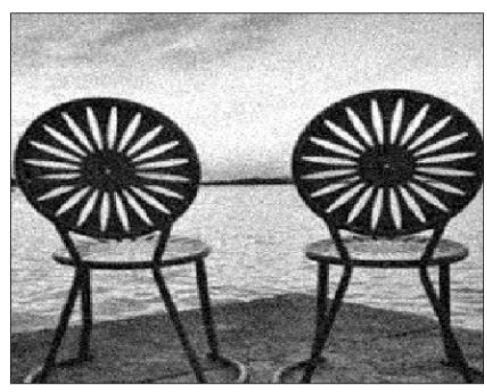

(c) $\sigma=15$

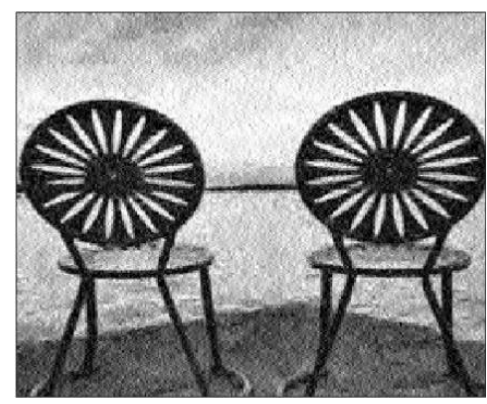

(e) $\sigma=25$

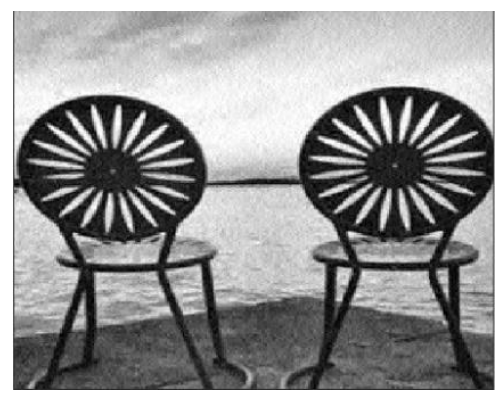

(b) $\sigma=10$

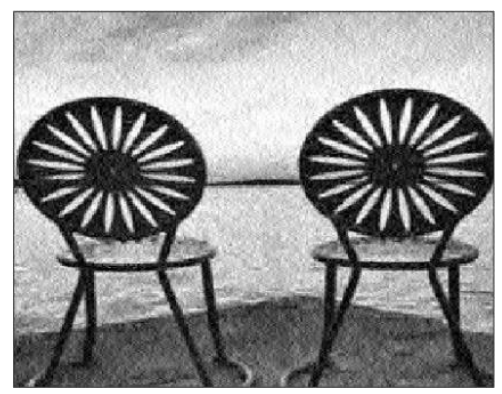

(d) $\sigma=20$

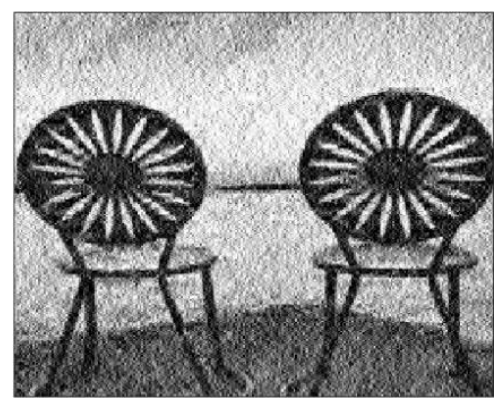

(f) $\sigma=50$

Figure 3

Distorted images by noise with different variances

It can be seen from Fig. 4 that even if the lowered VisuShrink threshold is used, the denoised images are getting notably blurred. When the proposed method with Otsu's threshold in this paper is used, smoother denoised images are obtained, and also more edge information is maintained. 


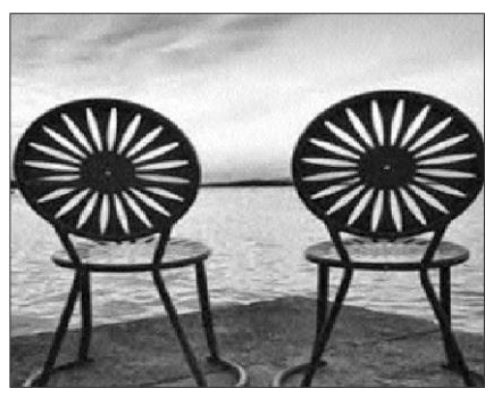

(a)

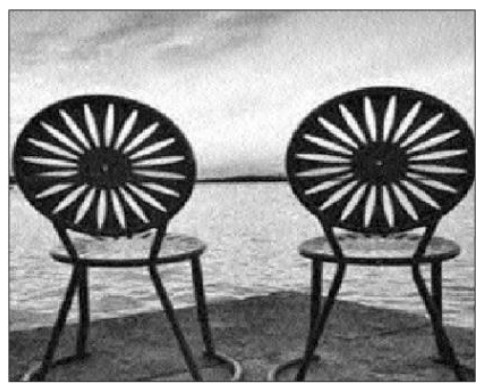

(c)

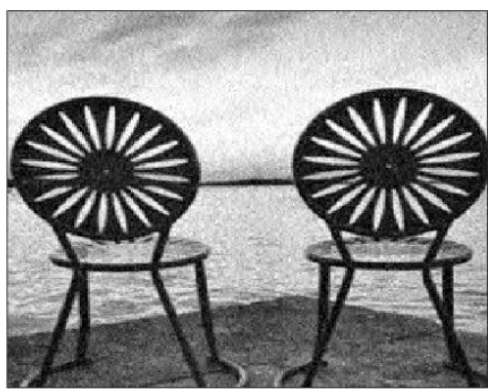

(e)

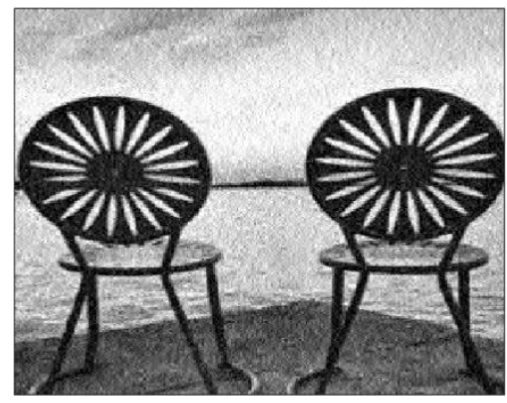

(g)

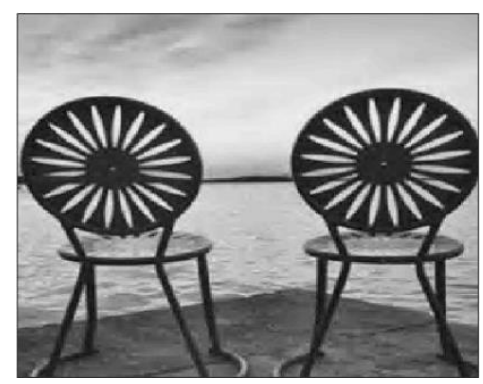

(b)

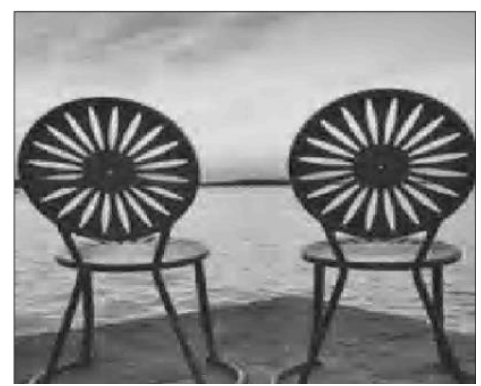

(d)

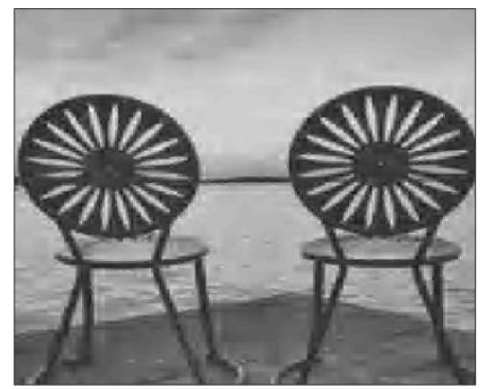

(f)

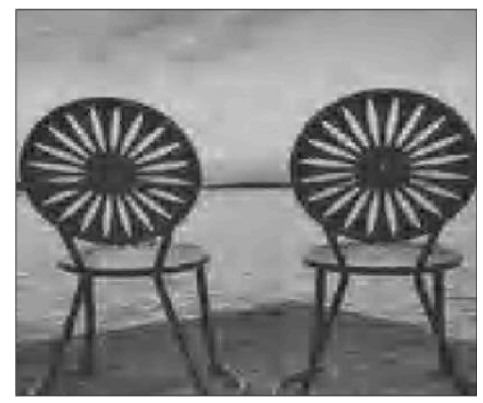

(h) 


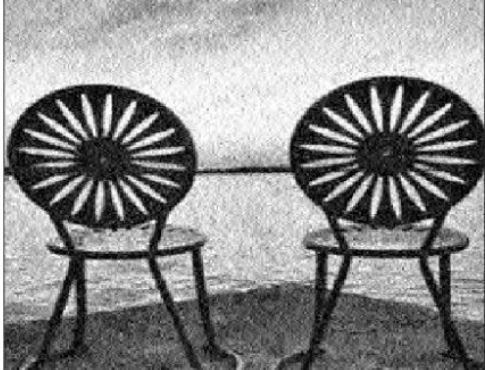

(1)

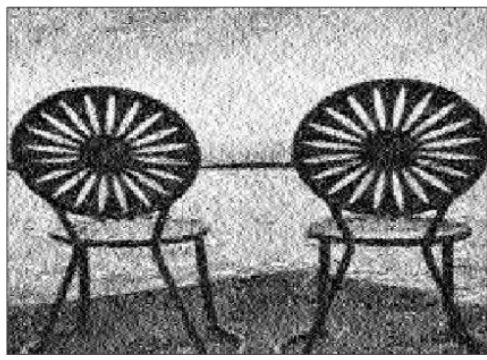

(k)

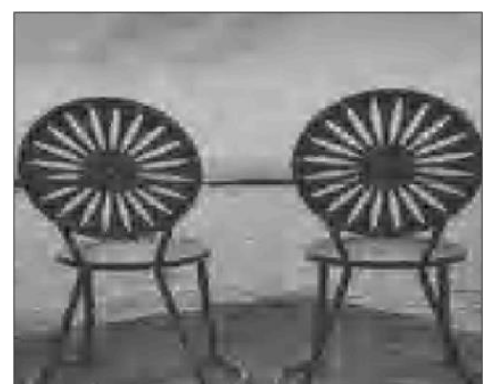

(j)

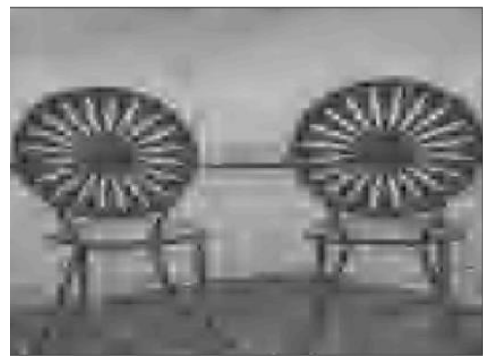

(1)

Figure 4

The obtained denoised images: (a),(c),(e),(g),(1),(k) are the results of the proposed method and (b),(d),(f),(h),(j),(l) are the results of the both VisuShrink and lowered VisuShrink thresholding with $\beta=0.2$

Table 2 presents the performance evaluation of the proposed method by comparing it with both VisuShrink and lowered VisuShrink thresholding according to the $P S N R$ value which is calculated as given in Eq. (10). From Table 2, it can be seen that there is an increase for PSNR up to 0.0656 1.1949 dB especially with higher noise variances for the proposed method in this paper. In addition, the dilemma of the determination of the threshold value in the classical denoising methods is overcome by using Otsu's thresholding value. In other respects, the problem of the determination of the noise variance in the Liu and Ma method presented in Ref. 21 has also been eliminated by this proposed method. The theoretical analysis and simulation results obtained in this paper indicate that the proposed method can protect more useful information of the image and also provide more denoised images compared with the commonly-used wavelet threshold denoising methods. Therefore, it can be concluded that the proposed threshold method denoising effect is better than other wavelet threshold denoising ones.

As given in Table 2, when the same design specifications and noise variances are used for Chairs image, the performance of the thresholding in the proposed method provides higher PSNR values and hence more qualified and denoised images. 
Table 2

Performance evaluation of the proposed method for Chairs image

$\begin{array}{lcc}\begin{array}{l}\text { Noise } \\ \text { variance }\end{array} & \begin{array}{c}\text { PSNR(dB) for the } \\ \text { proposed method }\end{array} & \begin{array}{c}\text { PSNR(dB) for Liu } \\ \text { and Ma method }\end{array} \\ \sigma=5 & 33.4257 & 33.3601 \\ \sigma=10 & 28.5888 & 27.9693 \\ \sigma=15 & 25.2984 & 24.9824 \\ \sigma=20 & 23.1415 & 22.8497 \\ \sigma=25 & 21.4797 & 20.2848 \\ \sigma=50 & 16.4644 & 15.3829\end{array}$

\section{Conclusions}

Image denoising is one of the most important applications in image processing. Using the knowledge that high frequencies characterize noise as well as edges, the denoising process and edge detection can be combined. Thus, deficiencies in commonly used denoising methods can be overcome. Although many denoising and edge detection methods are used today, different methods can be useful in different noise and image types. In this paper, a comprehensive framework for the image denoising method based on wavelet thresholding, by using Otsu's threshold, is provided. The appropriate adjustment factor for threshold value is determined by carrying out some trials for PSNR values and validation is done by applying the stages of the proposed method given in Fig. 1. Thereafter, a comparison between the proposed method and Liu and Ma [21] model, for the Chairs' image, is performed by using the same performance specifications. One can easily see from the obtained results that the denoising performance of the wavelet threshold denoising methods is effectively improved by the proposed method in this paper and it can be applied to different types of images. In the future additional research can be performed, relevant to this subject, by combining existing methods and then applied to different types of noise and images.

\section{References}

[1] G. Auber and P. Kolrnprobst: Mathematical Problems in Images Processing. Partial Differential Equations and the Calculus of Variations (2006) New York, NY: Springer Press

[2] A. K. Boyat and B. K. Joshi: "A review paper: noise models in digital image processing," Signal Image Process. Int. J. (SIPIJ). 6(2) (2015) 63-75

[3] L. Fan et al.: "Brief review of image denoising techniques," Vis. Comput. Ind. Biomed. Art. 2(7) (2019) 1-12

[4] B. Lee et al.: "Harmonic decomposition in PDE based de-noising technique for magnetic resonance electrical impedance tomography," Biomed. Eng. 52 (2005) 1912-1920 
[5] B. S. Mallat and W. L. Hwang: " Singularity detection and processing with wavelets," Inform. Theory 2 (1992) 617-643

[6] L. Zhu et al.: "Network-based method for mining novel HPV infection related genes using random walk with restart algorithm," Mol. Basis Dis. 6 (2017) 2376-2383

[7] M. Elad and M. Aharon: "Image De-noising via learned dictionaries and sparse representation," in Computer society Conference on Computer Vision and Pattern Recognition (New York, NY) (2006) 1-6

[8] N. M. Thanh and M. Chen: "Image denoising using adaptive Nneuro-fuzzy system," IAENG International Journal of Applied Mathematics 36(1) (2007) $1-7$

[9] C. F. Juang, Y. Y. Lin and R. B. Huang: " Dynamic system modelling using a recurrent interval-valued fuzzy neural network and its hardware implementation," Fuzzy Sets and Systems 179(1) (2011) 83-99

[10] W. Y. Cheng and C. F. Juang: "A fuzzy model with online incremental SVM and Margin-selective gradient descent learning for classifications problems," IEEE Transactions on Fuzzy Systems 22(2) (2014) 324-337

[11] C. Pozna and R. E. Precup: " Applications of signatures to expert systems modeling," Acta Polonica Hungarica 11(2) (2014) 21-39

[12] R. Zall and M. R. Kangavari: " On the construction of multi-relational classifier based on canonical correlation analysis," International Journal of Artifical Intelligence 17(2) (2019) 23-43

[13] H. Noh et al:: "Deep neural networks by noise: its interpretation and optimization," Machine Learn. 11 (2011) 1-10

[14] S. Swami et al.: "Regularizing deep networks using efficient layerwise adversarial training," arxiv:1705.07819 (2017)

[15] K. Khera and A. Saini: " Image denoising using KD-tree and nearest neighbour based kernel regression model," IJISET 2(7) (2015) 410-414

[16] W. Li, D. Kong and J. Wu: "A novel hybrid model based on extreme learning machine, k-nearest neighbor regression and wavelet denoising applied to short-term electric load forecasting," Energies 10 (2017) 1-16

[17] D. Liu et al.: "When image denoising meets high-level vision tasks: a deep learning approach," in Proc. of the IJCAI (2018) 842-848

[18] I. D. Borlea et al.: "A unified form of fuzzy C-means and K-means algorithms and its partitional implementation," Knowledge-Based Systems 214(2-3) (2021) 106731

[19] I. Johnstone and B. Silverman: "Empirical byes selection of wavelet thresholds," Annals Statist. 4 (2005) 1700-1752 
[20] H. Othman and S. E. Qian: "Noise reduction of hyper spectral imagery using hybrid spatial spectral derivative domain wavelet shrinkage," Geosci. Remote Sensin. 4 (2006) 397-408

[21] K. Bnou, S. Raghay and A. Hakim: " A wavelet denoising approach based onunsupervised learning model," EURASIP J. Adv. Sig. Pr. 36 (2020) 1-26

[22] D. L. Donoho and I. M. Johnstone: "Adapting to unknown wavelet shrinkage," J. Amer. Statist. Assoc. 90(432) (1995) 1200-1224

[23] P. Hedaoo and S. S. Godbole: "Wavelet based thresholding approach for image denoising," IJNSA 3(4) (2011) 16-21

[24] D. L. Donoho and I. M. Johnstone: "Ideal spatial adaptation by wavelet shrinkage," Biometrika 81(3) (1994) 425-455

[25] D. L. Donoho, "Denoising by soft-thresholding," IEEE Trans. Inf. Theory 41(3) (1995) 613-627

[26] F. Xiao and Y. Zhang: "A comparative study on thresholding methods in wavelet-based image denoising," Procedia Engineering 15 (2011) 3998-4003

[27] W. Liu and Z. Ma: "Wavelet image threshold denoising based on edge detection," in Proc. CESA (2006) 72-78

[28] M. Arora et al: "Wavelet denoising: comparative analysis and optimization using machine learning," in Proc. $20149^{\text {th }}$ International Conference on Industrial and Information Systems (ICIIS) (2014) 1-6

[29] S. Mallat and S. Zhong: "Characterization of signals from multiscale edges," IEEE Trans. Pattern Anal. Mach. Intell. 14(7) (1992) 710-733

[30] N. Otsu, "A threshold selection method from gray-level histograms," IEEE Trans. Syst. Man Cybern. Syst. 9 (1979) 62-6 